\title{
NONCOMMUTATIVE PRINCIPAL TORUS BUNDLES VIA PARAMETRISED STRICT DEFORMATION QUANTIZATION
}

\author{
KEITH C. HANNABUSS AND VARGHESE MATHAI
}

\begin{abstract}
In this paper, we initiate the study of a parametrised version of Rieffel's strict deformation quantization. We apply it to give a classification of noncommutative principal torus bundles, in terms of parametrised strict deformation quantization of ordinary principal torus bundles. The paper also contains a putative definition of noncommutative non-principal torus bundles.
\end{abstract}

\section{INTRODUCTION}

Operator theoretic deformation quantization appeared in quantum physics a long time ago, but was put on a firm footing relatively recently by Rieffel [15] (see the references therein), who called it strict deformation quantization, mainly to distinguish it from formal deformation quantization, where convergence isn't an issue. His theory has been remarkably successful, giving rise to many examples of noncommutative manifolds, which have become extremely useful both in mathematics and mathematical physics. In a recent paper [3] Echterhoff, Nest, and Oyono-Oyono defined noncommutative principal torus bundles, inspired by fundamental results in [17, as well as the T-duals of certain continuous trace algebras 13, 14. They also classified all noncommutative principal torus bundles in terms of (noncommutative) fibre products of principal torus bundles and group $\mathrm{C}^{*}$-algebras of lattices in simply-connected 2-step nilpotent Lie groups, cf. \$5. In this paper, we show that their classification can be neatly understood in terms of a generalization of Rieffel's strict deformation quantization [15, 16], to the parametrised case that is developed here. More precisely, we generalize to the parametrised case, the recent version of Rieffel's strict deformation quantization given by Kasprzak 9] based on work of Landstad [11, 12]. More precisely, we give a classification of noncommutative principal torus bundles, in terms of parametrised strict deformation quantization of ordinary principal torus bundles.

Strict deformation quantization theory works with smooth subalgebras, so we start with a section, $₫ \mathbb{1}$ on smooth subalgebras of $\mathrm{C}^{*}$-algebras and a smooth version of the noncommutative torus bundle theory. That is followed in $\$ 2$ by a summary recalling the ideas of Rieffel's strict deformation quantization, and then a section, 93. generalising that to a parametrised version. Then we explain in 4 . Kasprzak's recent account of Rieffel's strict deformation quantization theory based on ideas of Landstad, and extend it to a parametrised version. In $\$ 5$, after a summary of the

2000 Mathematics Subject Classification. 58B34 (81S10, 46L87, 16D90, 53D55).

Key words and phrases. Parametrised strict deformation quantization, Noncommutative principal torus bundles, T-duality.

Acknowledgements. V.M. thanks the Australian Research Council for support. 
relevant parts of the Echterhoff, Nest, and Oyono-Oyono classification of noncommutative principal torus bundles, we explain the connection to parametrised strict deformation quantization theory. We end with a section, 6 containing examples of parametrised strict deformation quantization including the case of principal torus bundles. It also contains a putative definition of noncommutative non-principal torus bundles. There is an appendix containing a discussion about factors of automorphy which is used in the paper.

\section{FibREWISE SMOOTH $*$-BUNDLES}

We begin by recalling the notion of $C^{*}$-bundles over $X$ and the special case of noncommutative principal bundles. Then we discuss the fibrewise smoothing of these, which is used in parametrised Rieffel deformation later on.

Let $X$ be a locally compact Hausdorff space and let $C_{0}(X)$ denote the $C^{*}$-algebra of continuous functions on $X$ that vanish at infinity. A $C^{*}$-bundle $A(X)$ over $X$ in the sense of $\left[3\right.$ is exactly a $C_{0}(X)$-algebra in the sense of Kasparov [8]. That is, $A(X)$ is a $C^{*}$-algebra together with a non-degenerate $*$-homomorphism

$$
\Phi_{A}: C_{0}(X) \rightarrow Z M(A(X)),
$$

called the structure map, where $Z M(A)$ denotes the center of the multiplier algebra $M(A)$ of $A$. The fibre over $x \in X$ is then $A(X)_{x}=A(X) / I_{x}$, where

$$
I_{x}=\left\{\Phi(f) \cdot a ; a \in A(X) \text { and } f \in C_{0}(X) \text { such that } f(x)=0\right\},
$$

and the canonical quotient map $q_{x}: A(X) \rightarrow A(X)_{x}$ is called the evaluation map at $x$.

Note that this definition does not require local triviality of the bundle, or even for the fibres of the bundle to be isomorphic to one another.

Let $G$ be a locally compact group. One says that there is a fibrewise action of $G$ on a $C^{*}$-bundle $A(X)$ if there is a homomorphism $\alpha: G \longrightarrow \operatorname{Aut}(A(X))$ which is $C_{0}(X)$-linear in the sense that

$$
\alpha_{g}(\Phi(f) a)=\Phi(f)\left(\alpha_{g}(a)\right), \quad \forall g \in G, a \in A(X), f \in C_{0}(X) .
$$

This means that $\alpha$ induces an action $\alpha^{x}$ on the fibre $A(X)_{x}$ for all $x \in X$.

The first observation is that if $A(X)$ is a $C^{*}$-algebra bundle over $X$ with a fibrewise action $\alpha$ of a Lie group $G$, then there is a canonical smooth *-algebra bundle over $X$. We recall its definition from [2]. A vector $y \in A(X)$ is said to be a smooth vector if the map

$$
G \ni g \longrightarrow \alpha_{g}(y) \in A(X)
$$

is a smooth map from $G$ to the normed vector space $A(X)$. Then

$$
\mathcal{A}^{\infty}(X)=\{y \in A(X) \mid y \text { is a smooth vector }\}
$$

is a *-subalgebra of $A(X)$ which is norm dense in $A(X)$. Since $G$ acts fibrewise on $A(X)$, it follows that $\mathcal{A}^{\infty}(X)$ is again a $C_{0}(X)$-algebra which is fibrewise smooth.

Let $T$ denote the torus of dimension $n$. The authors of $[3$ define a noncommutative principal T-bundle (or NCP T-bundle) over $X$ to be a separable $C^{*}$-bundle $A(X)$ together with a fibrewise action $\alpha: T \rightarrow \operatorname{Aut}(A(X))$ such that there is a Morita equivalence,

$$
A(X) \rtimes_{\alpha} T \cong C_{0}(X, \mathcal{K})
$$

as $C^{*}$-bundles over $X$, where $\mathcal{K}$ denotes the $C^{*}$-algebra of compact operators. 
The motivation for calling such $C^{*}$-bundles $A(X) \mathrm{NCP} T$-bundles arises from a special case of a theorem of Rieffel [17, which states that if $q: Y \longrightarrow X$ is a principal $T$-bundle, then $C_{0}(Y) \rtimes T$ is Morita equivalent to $C_{0}(X, \mathcal{K})$.

If $A(X)$ is a NCP $T$-bundle over $X$, then we call $\mathcal{A}^{\infty}(X)$ a fibrewise smooth noncommutative principal T-bundle (or fibrewise smooth NCP T-bundle) over X. In this paper, we are able to give a complete classification of fibrewise smooth NCP $T$-bundles over $X$ via a parametrised version of Rieffel's theory of strict deformation quantization.

\section{RiefFEl DEFORMATION}

Unlike Rieffel's deformation theory [15, 16, the version which we shall use 11, 12, 9] starts with multipliers, so in this section we shall recapitulate some standard results but in a formulation which suits the later extension to a parametrised theory and the Landstad-Kasprzak approach. In what follows, a Poisson bracket $\{$,$\} on$ $\mathcal{A}$ is a bilinear form from $\mathcal{A}$ to itself, which is a Hochschild 2-cocycle satisfying a couple of additional technical conditions that will not be repeated here, but we refer the reader to $\S 5$ in [15].

Definition $2.1(\S 5,[15])$. Let $\mathcal{A}$ be a dense $*$-subalgebra of a $\mathrm{C}^{*}$ algebra, equipped with a Poisson bracket $\{$,$\} . A strict deformation quantisation of \mathcal{A}$ in the direction of $\{$,$\} means an open interval I$ containing 0 in $\mathbf{R}$, together with associative products $\star_{\hbar}, \hbar \in I$, involutions and $\mathrm{C}^{*}$-norms on $\mathcal{A}$ which for $\hbar=0$ are the original product, involution and norm on $\mathcal{A}$, such that:

(1) The corresponding field of $\mathrm{C}^{*}$-algebras with continuity structure given by the elements of $\mathcal{A}$ as constant fields, is a continuous field of $\mathrm{C}^{*}$-algebras.

(2) For all $a, b \in \mathcal{A}$, as $\hbar \rightarrow 0$ one has $\left\|\left(a \star_{\hbar} b-a b\right) /(i \hbar)-\{a, b\}\right\| \rightarrow 0$.

Typically, one tries to find strict deformation quantizations of Poisson manifolds, thus obtaining interesting noncommutative manifolds.

Rieffel's definition and construction are motivated by Moyal's product but to link it with Kasprzak's work it is useful to give the background.

Suppose that $\mathcal{A}$ is a pre- $\mathrm{C}^{*}$-algebra with an action $\alpha$ of a locally compact abelian group $V$ (written additively), and let $\sigma$ be a multiplier on its Pontryagin dual $\widehat{V}$, that $\sigma: \widehat{V} \times \widehat{V} \rightarrow \mathbf{T}$ is a borel map, satisfying the cocycle identity

$$
\sigma(\xi, \eta) \sigma(\xi+\eta, \zeta)=\sigma(\xi, \eta+\zeta) \sigma(\eta, \zeta),
$$

for all $\xi, \eta, \zeta \in \widehat{V}$. The group of all such cocycles (or multipliers) is denoted by $Z^{2}(\widehat{V}, \mathbf{T})$. Two multipliers $\sigma_{1}$ and $\sigma_{2}$ are equivalent (or cohomologous) if and only if there is a borel map $\rho: \widehat{V} \rightarrow \mathbf{T}$ such that

$$
\sigma_{1}(\xi, \eta) \rho(\xi+\eta)=\sigma_{2}(\xi, \eta) \rho(\xi) \rho(\eta),
$$

and the equivalence classes form the cohomology group $H^{2}(\widehat{V}, \mathbf{T})$. A cocycle equivalent to the constant cocycle $\widehat{V} \times \widehat{V} \rightarrow\{1\}$ is said to be trivial.

Recalling that a bicharacter $\beta: V \times V \rightarrow \mathbf{T}$ defines characters $\beta_{\xi}^{1}: \eta \mapsto \beta(\xi, \eta)$ for each fixed $\xi$, and $\beta_{\eta}^{2}: \xi \mapsto \beta(\xi, \eta)$ for fixed $\eta$, we see that bicharacters always define cocycles, because

$$
\beta(\xi, \eta) \beta(\xi+\eta, \zeta)=\beta(\xi, \eta) \beta(\xi, \zeta) \beta(\eta, \zeta)=\beta(\xi, \eta+\zeta) \beta(\eta, \zeta) .
$$


Theorem 2.2 ([10, 5]). Every multiplier on an abelian group $\widehat{V}$ is equivalent to a bicharacter (and so is continuous). Two bicharacters $\beta_{1}$ and $\beta_{2}$ are equivalent if and only if $\beta=\beta_{1} \beta_{2}^{-1}$ a symmetric bicharacter, that is $\beta(\xi, \eta)=\beta(\eta, \xi)$. If $\widehat{V}=2 \widehat{V}$ then each cohomology class can be represented by a unique antisymmetric bicharacter $\beta$, that is $\beta(\xi, \eta)=\beta(\eta, \xi)^{-1}$.

We can therefore assume that $\sigma$ is a bicharacter, and this means that it is actually continuous in each variable. When $\widehat{V}=2 \widehat{V}$, the element $\sigma\left(\xi, \frac{1}{2} \eta\right) / \sigma\left(\eta, \frac{1}{2} \xi\right)$ gives the canonical antisymmetric bicharacter representative of the class containing $\sigma$. We note that vector groups $\widehat{V}=\mathbf{R}^{n}=2 \widehat{V}$, so that each cocycle can be represented by a continuous antisymmetric antisymmetric bicharacter, which must be the exponential $\exp [i \pi s(\xi, \eta)]$ of a skew-symmetric bilinear form $s$. These can be identified with $\bigwedge^{2} V$. There is a similar analysis for lattices $L \cong \mathbf{Z}^{n}$, where the bicharacters are given by the torus $\bigwedge^{2}(V / L)=\bigwedge^{2} V / \bigwedge^{2} L$ (restrictions modulo those with trivial restriction), but a torus $\mathbf{T}^{n}$ has only trivial bicharacters $\beta(\xi, \eta)=1$, due to the following observation.

Corollary 2.3. There are no non-trivial bicharacters on a connected compact group $V$.

This follows because the non-trivial multipliers on the dual of an infinite connected compact group (such as $V=\mathbf{T}^{2 n}$ ) are never invertible, since the dual (e.g. $\widehat{V}=\mathbf{Z}^{2 n}$ ) is discrete and the two groups are not isomorphic.

Theorem 2.4. Given a continuous bicharacter cocycle $\sigma$ on $\widehat{V}$ and a pre-C $C^{*}$ algebra $\mathcal{A}$ we may form the *-algebra of functions $f, g: \widehat{V} \rightarrow \mathcal{A}$ smooth with respect to the translation automorphisms $\tau_{u}[f]=f(u+v)$, with the twisted convolution product

$$
(f * g)(\xi)=\int_{\widehat{V}} \sigma(\eta, \xi-\eta) f(\eta) g(\xi-\eta) d \eta,
$$

and involution $f^{*}(\xi)=\sigma(\xi, \xi) \overline{f(-\xi)}$. Up to isomorphism this algebra depends only on the cohomology class of $\sigma$.

Proof. The cocycle identity on $\sigma$ ensures associativity. When $\sigma$ is an antisymmetric bicharacter the involution reduces to $f^{*}(\xi)=\overline{f(-\xi)}$. Changing $\sigma$ to

$$
\sigma(\xi, \eta) \rho(\xi) \rho(\eta) \rho(\xi+\eta)^{-1}
$$

gives the algebra isomorphism $f \mapsto \rho . f$ (the pointwise product).

These functions can be Fourier transformed to functions on $V$

$$
\widehat{f}(v)=\int_{\widehat{V}} \xi(v) f(\xi) d \xi,
$$

where we assume that the Haar measure is normalised to make the transform unitary, and one has the usual inverse transform.

Theorem 2.5. The transformed product is

$$
\widehat{(f * g)}(v)=\int \sigma(\eta, \xi) \overline{\eta(u) \xi(w)} \widehat{f}(v+u) \widehat{g}(v+w) d u d w d \xi d \eta
$$


Proof. We calculate that

$$
\begin{aligned}
\widehat{(f * g)}(v) & =\int_{\widehat{V}} \xi(v)(f \star g)(\xi) d \xi \\
& =\int_{\widehat{V} \times \widehat{V}} \xi(v) \sigma\left(\eta, \xi \eta^{-1}\right) f(\eta) g\left(\xi \eta^{-1}\right) d \xi d \eta \\
& =\int_{\widehat{V} \times \widehat{V}} \sigma(\eta, \xi) \eta(v) f(\eta) \xi(v) g(\xi) d \xi d \eta \\
& =\int \sigma(\eta, \xi) \eta(v) \widehat{f}(u) \overline{\eta(u)} \xi(v) \widehat{g}(w) \overline{\xi(w)} d u d w d \xi d \eta \\
& =\int \sigma(\eta, \xi) \overline{\eta(u) \xi(w)} \widehat{f}(v+u) \widehat{g}(v+w) d u d w d \xi d \eta
\end{aligned}
$$

where we replaced $u$ and $w$ by $v+u$ and $v+w$ in the last step.

We now want to connect this transformed product with Rieffel's deformation. To this end we introduce a bicharacter $e$ on $V$, which defines a homomorphism $e^{1}: V \rightarrow \widehat{V}$. Rieffel works with a vector group $V$ and $e(u, w)=\exp (i(u \cdot w))$ for some inner product on $V$. When $\sigma$ is non-degenerate (that is, $\sigma^{1}: \widehat{V} \rightarrow V$ is an isomorphism) we can choose $e$ so that $e^{1}$ is the inverse of $\sigma^{1}$, but in general we have an automorphism $T=\sigma^{1} \circ e^{1}: V \rightarrow V$. As a final piece of notation we introduce the adjoint $T^{*}$ with respect to $e: e\left(T^{*} u, w\right)=e(u, T w)$.

Proposition 2.6. The bicharacters $\sigma$ and e are related by $\sigma\left(e_{u}^{1}, e_{v}^{1}\right)=e_{v}^{1}(T u)=$ $e(T u, v)$ for all $u, v \in V$. Suppose that $\sigma$ is an antisymmetric bicharacter. Then if $e$ is symmetric $T=-T^{*}$, and if $e$ is antisymmetric $T=T^{*}$.

Proof. By definition we have

$$
\sigma\left(e_{u}^{1}, e_{v}^{1}\right)=e_{v}^{1}(T u)=e(T u, v) .
$$

Since $\sigma$ is skew symmetric this gives

$$
e(T u, v)=\sigma\left(e_{u}^{1}, e_{v}^{1}\right)=\sigma\left(e_{v}^{1}, e_{u}^{1}\right)^{-1}=e(T v, u)^{-1}=e(-T v, u) .
$$

When $e$ is symmetric this shows that $e(T u, v)=e(u,-T v)$, so that $T=-T^{*}$, and when $e$ is antisymmetric $T=T^{*}$.

Theorem 2.7. Given a non-degenerate bicharacter e on $V$, set $T=\sigma^{1} \circ e^{1}: V \rightarrow$ $V$. and $e\left(T^{*} u, w\right)=e(u, T w)$. Then

$$
\widehat{(f * g)}(v)=\int e(u, w) \widehat{f}\left(v+T^{*} u\right) \widehat{g}(v+w) d u d w .
$$

We change the order of integration in our earlier expression for $f * g$ and concentrate on the integrals over $\widehat{V}$ :

$$
\int_{\widehat{V} \times \widehat{V}} \sigma(\eta, \xi) \overline{\eta(u) \xi(w)} d \xi d \eta
$$

Since $e$ is nondegenerate we may set $\eta=e_{v}$, and then, by definition, we have

$$
\sigma\left(e_{v}, \xi\right) \overline{e_{v}(u) \xi(w)}=\xi(T v) \overline{e(v, u) \xi(w)}
$$


By the Fourier inversion theorem, integration over $\xi$ gives a delta function $\delta(T v-w)$. Replacing $u$ by $T^{*} u$ and integrating over $v$, we now get

$$
\int \sigma\left(e_{v}, \xi\right) \overline{e\left(v, T^{*} u\right) \xi(w)} d \xi d v=\int \delta(w-T v) e(T v, u) d v=e(w, u),
$$

Up to a multiple, integration over $\eta$ and $v$ are the same, and with appropriate choices of measure we can ensure that they agree precisely. Then inserting this into the original formula for the product we have

$$
\widehat{(f * g)}(v)=\int e(u, w) \widehat{f}\left(v+T^{*} u\right) \widehat{g}(v+w) d u d w .
$$

Theorem 2.8. The Fourier transformed product $\widehat{(f * g)}=\widehat{f} \star \widehat{g}$ where

$$
(\widehat{f} \star \widehat{g})(v)=\int e(u, w) \widehat{f}\left(v+T^{*} u\right) \widehat{g}(v+w) d u d w .
$$

In terms of the translation automorphisms $\tau_{w}[g](v)=g(v+w)$, we have

$$
(\widehat{f} \star \widehat{g})(v)=\int e(u, w) \tau_{T^{*} u}[\widehat{f}](v) \tau_{w}[\widehat{g}](v) d u d w .
$$

Evaluating at the identity $v=0$ gives

$$
(\widehat{f} \star \widehat{g})(0)=\int e(u, w) \tau_{T^{*} u}[\widehat{f}](0) \tau_{w}[\widehat{g}](0) d u d w .
$$

Rieffel noticed that this formula can now be interpreted whenever $\alpha$ defines automorphisms of $\mathcal{A}$, so that one can define

$$
a \star b=\int e(u, w) \alpha_{T^{*} u}[a] \alpha_{w}[b] d u d w
$$

for $a$ and $b$ in the algebra. (Our $T^{*}$ is Rieffel's $J$.) When both bicharacters $\sigma$ and $e$ are nondegenerate we can also write this as

$$
a \star b=\int \operatorname{det}\left[T^{*}\right]^{-1} e\left(T^{*-1} u, w\right) \alpha_{u}[a] \alpha_{w}[b] d u d w .
$$

The above arguments are formal and one must check that the integrals converge. In the standard Moyal theory this is done by working only with Schwarz functions and in the general case one uses the smooth vectors $\mathcal{A}^{\infty}$ for the action $\alpha$, which form a dense Fréchet subalgebra of $\mathcal{A}$.

For vector groups this works particularly smoothly, and one obtains a strict deformation quantisation [15, Theorem 9.3. However, there are technical problems when $V=\mathbf{T}^{2 n}$ since, as we have seen, there are no nontrivial bicharacters $e$ on $V$. There are two ways of dealing with this problem. One is by the Kasprzak-Landstad approach of working with the dual crossed product algebra, [12, 9, and the other is Rieffel's approach of lifting the action of the torus $T=V / L$ (with $L$ a lattice), to the vector group $V, 15$, Ch 2 .

\section{Parametrised Rieffel Deformations}

An interesting generalisation comes from inserting a parameter. More precisely, we work with a $C_{0}(X)$-algebra $\mathcal{A}$, where $X$ is a locally compact Hausdorff space. (That is there is a map $C_{0}(X) \rightarrow \mathcal{Z} M \mathcal{A}$.) Consider a function $\sigma \in C_{b}\left(X, Z^{2}(\widehat{V}, \mathbf{T})\right.$ ) taking values in the bicharacter cocycles. At each point $x \in X$ this defines a multiplier $\sigma_{x}$, and a map $\sigma_{x}^{1}: \widehat{V} \rightarrow V$. We then form $T_{x}=\sigma_{x}^{1} \circ e^{1}$ and its adoint $T_{x}^{*}$ 
with respect to $e$, where $e, e^{1}$ are defined just prior to Proposition 2.6 If the image of $\sigma$ lies in the non-degenerate cocycles we can then form the continuous function $x \mapsto e\left(T_{x}^{*-1} u, v\right)$, which acts on $\mathcal{A}$.

Theorem 3.1. Given a $C_{0}(X)$-algebra $\mathcal{A}$, where $X$ is a locally compact Hausdorff space, and a function $\sigma \in C_{b}\left(X, Z^{2}(\widehat{V}, \mathbf{T})\right)$ taking values in the nondegenerate bicharacter cocycles, let $T_{x}=\sigma_{x}^{1} \circ e^{1}$ and $e\left(T_{x}^{*} u, w\right)=e\left(u, T_{x} w\right)$. Then, if $T$ has an inverse in a subalgebra of $C_{b}(X)$ whose action preserves the (fibrewise) smooth subalgebra $\mathcal{A}^{\infty}$, one has a product

$$
a \star b=\int \operatorname{det}\left[T^{*}\right]^{-1} e\left(T^{*-1} u, w\right) \alpha_{u}[a] \alpha_{w}[b] d u d w,
$$

defined by the actions of the continuous functions $\operatorname{det}\left[T^{*}\right]^{-1}$ and $e\left(T^{*-1} u, w\right)$ on $\mathcal{A}$. This gives an algebra $\mathcal{A}_{\sigma}$ with an involution, which inherits a $C_{0}(X)$-algebra structure. (The $C_{0}(X)$-structure on the algebra is such that for $F \in C_{0}(X)$ we have $F .(a \star b)=(F . a) \star b=a \star(F . b)$.

For vector groups it follows from the definition that the iterated parametrised strict deformation quantization $\left(\mathcal{A}_{\sigma_{1}}\right)_{\sigma_{2}} \cong \mathcal{A}_{\sigma_{1} \sigma_{2}}$, with the isomorphism defined by the obvious identification map. This follows on writing down the repeated deformation product and evaluating a double integral using Parseval's formula or the Fourier inversion formula. Alternatively we can note that the bicharacter $\sigma^{-1}$ can always be written in Rieffel form, and then the result follows from his. Yet another approach would be to use the equivalence with Kasprzak's formulation given below, and then to deduce it from his result. In particular, we can undeform $\mathcal{A}_{\sigma}$ using $\bar{\sigma}$.

In this more general context we can generalise Rieffel's discussion of the action of continuous automorphisms of the group $V$ (which give $G L(V)$ when $V$ is a vector group), to allow functions $S \in C^{\infty}(X, A u t(V))$ and using

$$
a \star_{S} b=\int_{V \times V} \sigma^{-1}(S u, S w) \cdot\left(\alpha_{u}[a] \alpha_{w}[b]\right) d u d w .
$$

Note that the original automorphisms of $V$ on $\mathcal{A}$ are also automorphisms of the deformed algebra, since

$$
\begin{aligned}
\alpha_{v}[a] \star_{\sigma} \alpha_{v}[b] & =\int_{V \times V} \operatorname{det}\left[T^{*}\right]^{-1} e\left(T^{*-1} u, v\right) \cdot\left(\alpha_{u+v}[a] \alpha_{w+v}[b]\right) d u d w \\
& =\int_{V \times V} \operatorname{det}\left[T^{*}\right]^{-1} e\left(T^{*-1} u, v\right) \cdot \alpha_{v}\left[\left(\alpha_{u}[a] \alpha_{w}[b]\right) d u d w\right. \\
& =\alpha_{v}\left[a \star_{\sigma} b\right]
\end{aligned}
$$

since $\alpha_{w}$ commutes with the $C_{0}(X)$ action.

We constructed the deformation as the dual of a twisted crossed product, and the reverse is also true. Given an algebra $\mathcal{A}$ with an action of $V$ one can take the crossed product $\mathcal{A} \rtimes V$ with a dual action of $\widehat{V}$. Looking first at the unparametrised case, when $\sigma$ is non-degenerate there is a dual multiplier $\widehat{\sigma}$ on $V$ defined by $\widehat{\sigma}\left(u, \sigma_{1} \eta\right)=$ $\eta(u)$, and similarly for $\widehat{e}\left(\xi, e^{1} v\right)=\xi(v)$, and $\widehat{T}=\widehat{\sigma}^{1} \circ \widehat{e}^{1}$. These definitions effectively mean that $\hat{e}^{1}$ is the inverse of $e^{1}$ and similarly for $\sigma$. We can now deform the crossed 
product.

$$
\begin{aligned}
\left(a \star_{\widehat{\sigma}} b\right)(v) & =\int \widehat{e}(\xi, \eta) \widehat{\alpha}_{\widehat{T} \xi}[a] \widehat{\alpha}_{\eta}[b] d \xi d \eta \\
& =\int \widehat{e}(\xi, \eta) \widehat{\alpha}_{\widehat{T} \xi}[a](u) \alpha_{u}\left[\widehat{\alpha}_{\eta}[b](v-u)\right] d \xi d \eta d u \\
& =\int \widehat{e}(\xi, \eta)(\widehat{T} \xi)(u) \eta(v-u) a(u) \alpha_{u}[b(v-u)] d \xi d \eta d u \\
& \left.=\int \eta\left(\widehat{e}^{1} \xi\right) \widehat{T} \xi\right)(u) \eta(v-u) a(u) \alpha_{u}[b(v-u)] d \xi d \eta d u
\end{aligned}
$$

The integral over $\eta$ gives a delta function concentrated on $\widehat{e}^{1} \xi=u-v$, or equivalently where $\xi=e^{1}(u-v)$, so that the $\xi$ integral then gives

$$
\left(a \star_{\widehat{\sigma}} b\right)(v)=\int\left(\widehat{T} e^{1}(u-v)\right)(u) a(u) \alpha_{u}[b(v-u)], d u .
$$

By definition, we have

$$
\widehat{T} e^{1}=\widehat{\sigma}^{1} \widehat{e}^{1} e^{1}=\widehat{\sigma}^{1},
$$

which leads to the reduction

$\left(a \star_{\widehat{\sigma}} b\right)(v)=\int\left(\widehat{\sigma}^{1}(u-v)\right)(u) a(u) \alpha_{u}[b(v-u)], d u=\int \widehat{\sigma}(u-v, u) a(u) \alpha_{u}[b(v-u)], d u$.

This is a twisted crossed product with multiplier. There is a similar parametrised version.

\section{LANDSTAD-KASPRZAK AND RIEFFEL DEFORMATION}

Building on work of Landstad [11, 12, Kasprzak 9] gives an alternative dual picture of deformation theory. It is useful to give the equivalence with Rieffel deformation explicitly, as Kasprzak omits the details. (The correspondence is not obvious since the algebra elements in Rieffel's deformation are the same and only the product changes, whereas in Kasprzak's formulation the deformed and undeformed algebras are distinct fixed point subalgebras of the multiplier algebra of the crossed product, with different actions of $V$. Smoothness or some equivalent is also needed; Landstad suggests in 12 that it is sufficient to use the Fourier algebra instead of smooth subalgebras.) In the following account we use Rieffel's notation of $\alpha$ rather than $\rho$ for the automorphisms.

Landstad showed in 11] that when a group $V$ acts on an algebra $\mathcal{A}$, the crossed product $\mathcal{B}=\mathcal{A} \rtimes V$ has a coaction which is defined by a homomorphism $\lambda: V \rightarrow$ $\mathcal{U} M B$ (the unitary multiplier algebra). By integration $\lambda$ extends to $C(V) \rightarrow \mathcal{M B}$. When $V$ is abelian there is also the dual Takai-Takesaki action $\widehat{\alpha}$ of $\widehat{V}$, and these interact by $\widehat{\alpha}_{\xi}\left[\lambda_{v}\right]=\xi(v) \lambda_{v}$. By Takai-Takesaki duality $\mathcal{B} \rtimes_{\widehat{\alpha}} \widehat{V}$ is isomorphic to $\mathcal{A} \otimes \mathcal{K}$, reconstructing $\mathcal{A}$ up to stable equivalence. When $\mathcal{B}$ has the Landstad $\lambda$ as well we can deduce a stronger duality that there is an algebra $\mathcal{A}$ with $V$-action $\alpha$ such that $\mathcal{B}=\mathcal{A} \rtimes_{\alpha} V$. Kasprzak's idea is that $\widehat{\alpha}_{\xi}$ can be deformed by a cocycle $\sigma$ for $\widehat{V}$ to a new action $\widehat{\alpha}_{\xi}^{\sigma}$.

Theorem 4.1. 9]. Let $(\mathcal{B}, \lambda, \widehat{\alpha})$ be as above, and $\sigma$ a continuous cocycle for $\widehat{V}$. Setting $U_{\xi}=\lambda\left(\sigma_{\xi}^{1}\right)$ there is an action of $\widehat{V}$ on $\mathcal{A}$ given by

$$
\widehat{\alpha}_{\xi}^{\sigma}: b \mapsto U_{x} i^{*} \widehat{\alpha}_{\xi}[b] U_{\xi},
$$


which also satisfies $\alpha_{\xi}^{\sigma}\left[\lambda_{v}\right]=\xi(v) \lambda_{v}$.

Corollary 4.2. There is an algebra $\mathcal{A}^{\sigma}$ and $V$-action $\alpha^{\sigma}$ such that the crossed product $\mathcal{A}^{\sigma} \rtimes_{\alpha^{\sigma}} V \cong \mathcal{B}=\mathcal{A} \rtimes_{\alpha} V$.

The deformed and undeformed algebras can be identified with the subalgebras of $\mathcal{M} B$ fixed by the action $\widehat{\alpha}$ of $\widehat{V}$.

In particular, the undeformed algebra is fixed under the dual group action on the crossed product given by $\widehat{\alpha}_{\xi}[a](v)=\xi(v) a(v)$. The fixed points of this action are distributions concentrated on the group identity $v=0$, which make sense as elements of the multiplier algebra. They give an algebra isomorphic to $\mathcal{A}$, and this is just Rieffel's construction as defined above.

For the algebra deformed by $\sigma, U_{\xi}=\delta_{\sigma_{\xi}^{1}}$, and by the covariance property of crossed products the adjoint action of $U_{\xi}$ is the same as the action of $\alpha_{\sigma_{\xi}^{1}}$. We therefore have

$$
\left.\widehat{\alpha}_{\xi}^{\sigma}[a](v)=\alpha_{\sigma_{\xi}^{1}}^{-1}\left[\widehat{\alpha}_{\xi}\right)[a(v)]\right]=\xi(x) \alpha_{\sigma_{\xi}^{1}}^{-1}[a(v)]
$$

Changing variable, the fixed subalgebra, where $\widehat{\alpha}_{\xi}^{\sigma}[a]=a$, therefore consists of elements $a$ satisfying

$$
\alpha_{\sigma_{\xi}^{1}}[a(v)]=\xi(v) a(v) .
$$

In the notation of previous sections we set $\xi=e_{u}^{1}$ so that $\sigma_{\xi}^{1}=T u$, and then the condition becomes

$$
\alpha_{T u}[a(v)]=e(u, v) a(v) .
$$

Thus the value of $a(v)$ always lies in a particular eigenspace of the action $\alpha$. (In particular, when $e$ is an antisymmetric bicharacter $a(0)$ must be in the fixed point algebra of $\alpha_{T}$.) In other words we can characterise the fixed point algebra elements as the elements whose value at $v$ lies in the relevant spectral subspace $\operatorname{ker}\left[\alpha_{T u}-\right.$ $e(u, v)]$ of the action of $\alpha$.

To get all the eigenspaces we must do a direct integral, or, for suitably wellbehaved functions (the smooth subalgebra), we set $I(a)=\int a(v) d v$.

Theorem 4.3. When $T$ is invertible, the product of fixed point algebra elements a and $b$ satisfies

$$
I(a * b)=I(a) \star I(b) .
$$

Proof. When $T$ is invertible, the product of fixed point algebra elements is given by

$$
\begin{aligned}
I(a * b) & =\int a(u) \alpha_{u}[b(v-u)] d u d v \\
& =\int a(u) \alpha_{u}[b(v)] d u d v \\
& =\int e\left(T^{-1} u, v\right) a(u) b(v) d u d v .
\end{aligned}
$$


On the other hand, under the same conditions, and with $e$ symmetric, so that $T=-T^{*}$

$$
\begin{aligned}
(I(a) \star I(b)) & =\int \operatorname{det}\left[T^{*}\right]^{-1} e\left(T^{*-1} u, v\right) \alpha_{u}[I(a)] \alpha_{v}[I(b)] d u d v \\
& =\int \operatorname{det}\left[T^{*}\right]^{-1} e\left(-T^{-1} u, v\right) \alpha_{u}[a(y)] \alpha_{v}[b(x)] d x d y d u d v \\
& =\int \operatorname{det}\left[T^{*}\right]^{-1} e\left(T^{-1} u,-v\right) e\left(T^{-1} u, y\right) a(y) e\left(T^{-1} v, x\right) b(x) d x d y d u d v \\
& =\int \operatorname{det}\left[T^{*}\right]^{-1} e\left(T^{-1} u, y-v\right) a(y) e\left(T^{-1} v, x\right) b(x) d x d y d u d v
\end{aligned}
$$

The integral of $e\left(T^{*-1} u, v-y\right)$ over $u$ produces a delta function concentrated on $v=-y$, and then the $v$ integral gives

$$
\begin{aligned}
(I(a) \star I(b)) & =\int e\left(T^{-1} y, x\right) a(y) b(x) d u d v \\
& =\int e\left(T^{-1} x, y\right) a(y) b(x) d u d v \\
& =I(a * b),
\end{aligned}
$$

showing that $I$ defines a homomorphism from the Kasprzak deformation to the Rieffel deformation.

Standard harmonic analysis shows that this is formally an isomorphism on suitably defined smooth subalgebras. (The inverse map takes an algebra element $a$ and does harmonic analysis of $\alpha$ action setting $a(x)$ to be the component of $a$ such that $\alpha_{y}[a(x)]=\sigma^{-1}(x, y) a(x)$.) The same constructions can be carried out for $C_{0}(X)$-algebras.

\section{Classifying noncommutative principal torus Bundles}

The noncommutative principal torus bundles of Echterhoff, Nest, and OyonoOyono, whose definition was recalled in Section 1, were classified in [3] and will be outlined in this section. We also give a classification of fibrewise smooth noncommutative principal torus bundles in terms of parametrized strict deformation quantization of ordinary principal torus bundles.

By Takai-Takesaki duality $\mathcal{A}(X)$ is Morita equivalent to $C_{0}(X, \mathcal{K}) \rtimes \widehat{T}$, so the authors in [3] note that the NCPT-bundles can be classified by up to Morita equivalence by the outer equivalence classes $\mathcal{E}_{\widehat{T}}(X)$ of $\widehat{T}$-actions, and one has the sequence

$$
0 \longrightarrow H^{1}(X, T) \longrightarrow \mathcal{E}_{\widehat{T}}(X) \longrightarrow C\left(X, H^{2}(\widehat{T}, \mathbf{T})\right) \longrightarrow 0 .
$$

This leads to a classification in terms of a principal torus bundle $q: Y \rightarrow X$, from $H^{1}(X, T)$, and a map $\sigma \in C_{b}\left(X, H^{2}(\widehat{T}, \mathbf{T})\right)$, the equivalence classes of multipliers on the dual group $\widehat{T}$. These data define a noncommutative torus bundle by forming the fixed point algebra

$$
\left.\left[C_{0}(Y) \otimes_{C_{0}(\widehat{Z})} C^{*}\left(H_{\sigma}\right)\right)\right]^{T}
$$

with $C^{*}\left(H_{\sigma}\right)$ being the bundle of group $C^{*}$-algebras of the central extensions of $\widehat{T}$ by $\widehat{Z}:=H^{2}(\widehat{T}, \mathbf{T})$ defined by $\sigma(x)$ at $x$, the action of $C_{0}(\widehat{Z})$ on $C_{0}(Y)$ coming from 
the composition $\sigma \circ q: Y \rightarrow X \rightarrow \widehat{Z}$ and that on $C^{*}\left(H_{\sigma}\right)$ from the natural action of a subgroup algebra. The bundle is a classical principal bundle when $\sigma$ is the constant map to the trivial multiplier 1 (or indeed is homotopic to any constant map).

A key observation is that the datum $\sigma$, or more practically an equivalence class of $\sigma \in C\left(X, Z^{2}(\widehat{T}, \mathbf{T})\right)$ can be identified with the similar map in the parametrised deformation theory, and that the Landstad-Kasprzak dual deformation theory conveniently matches the duality in the definition of NCPT-bundles with the group $V=T$ and the dual algebra $\mathcal{B}=C_{0}(X, \mathcal{K})$. The analysis in 3 starts with the case of $X$ a point, where the algebra is shown to be the twisted $\mathrm{C}^{*}$ group algebra of $\widehat{T}$ defined by the multiplier $\sigma$, or, equivalently, the deformed algebra defined by $\sigma$. The same construction can be carried out in the case of general $X$ using our parametrised deformation constructions, and this can then be twisted using an ordinary principal $T$-bundle. Now given a fibrewise smooth NCPT-bundle $\mathcal{A}^{\infty}(X)$ the defining deformation $\sigma$ can be removed by a further deformation by $\bar{\sigma}$ since then one has a total deformation $\sigma \bar{\sigma}=1$, and a constant map 1 gives an ordinary principal torus bundle up to $T$-equivariant Morita equivalence over $C_{0}(X)$. In other words one can recover the principal torus bundle $q: Y \rightarrow X$ in this way up to $T$-equivariant Morita equivalence over $C_{0}(X)$ via an iterated parametrized strict deformation quantization. To summarize, we have the following main result, which follows from Theorem 3.1 \&4, Example 6.2 and the observations above.

Theorem 5.1. Given a fibrewise smooth NCPT-bundle $\mathcal{A}^{\infty}(X)$, there is a defining deformation $\sigma \in C_{b}\left(X, Z^{2}(\widehat{T}, \mathbf{T})\right)$ and a principal torus bundle $q: Y \rightarrow X$ such that $\mathcal{A}^{\infty}(X)$ is T-equivariant Morita equivalent over $C_{0}(X)$, to the parametrised strict deformation quantization of $C_{\text {fibre }}^{\infty}(Y)$ (continuous functions on $Y$ that are fibrewise smooth) with respect to $\sigma$, that is,

$$
\mathcal{A}^{\infty}(X) \cong C_{\text {fibre }}^{\infty}(Y)_{\sigma} .
$$

Conversely, by Example 6.2, the parametrised strict deformation quantization of $C_{\mathrm{fibre}}^{\infty}(Y)$ is the noncommutative principal torus bundle $C_{\mathrm{fibre}}^{\infty}(Y)_{\sigma}$.

\section{Fine Structure of PARAmetrised Strict Deformation QUANTIZATION}

We have seen in Theorem 5.1 that all fibrewise smooth NCPT-bundles are just parametrised strict deformation quantizations of ordinary principal torus bundles. We will use this to write out the fine structure of fibrewise smooth NCPT-bundles.

Example 6.1. We begin by recalling the construction by Rieffel [15] realizing the smooth noncommutative torus as a deformation quantization of the smooth functions on a torus $T=\mathbf{R}^{n} / \mathbf{Z}^{n}$ of dimension equal to $n$.

Recall that any translation invariant Poisson bracket on $T$ is just

$$
\{a, b\}=\sum \theta_{i j} \frac{\partial a}{\partial x_{i}} \frac{\partial b}{\partial x_{j}},
$$

for $a, b \in C^{\infty}(T)$, where $\left(\theta_{i j}\right)$ is a skew symmetric matrix. The action of $T$ on itself is given by translation. The Fourier transform is an isomorphism between $C^{\infty}(T)$ and $\mathcal{S}(\hat{T})$, taking the pointwise product on $C^{\infty}(T)$ to the convolution product on $\mathcal{S}(\hat{T})$ and taking differentiation with respect to a coordinate function to multiplication 
by the dual coordinate. In particular, the Fourier transform of the Poisson bracket gives rise to an operation on $\mathcal{S}(\hat{T})$ denoted the same. For $\phi, \psi \in \mathcal{S}(\hat{T})$, define

$$
\{\psi, \phi\}(p)=-4 \pi^{2} \sum_{p_{1}+p_{2}=p} \psi\left(p_{1}\right) \phi\left(p_{2}\right) \gamma\left(p_{1}, p_{2}\right)
$$

where $\gamma$ is the skew symmetric form on $\hat{T}$ defined by

$$
\gamma\left(p_{1}, p_{2}\right)=\sum \theta_{i j} p_{1, i} p_{2, j} .
$$

For $\hbar \in \mathbf{R}$, define a skew bicharacter $\sigma_{\hbar}$ on $\hat{T}$ by

$$
\sigma_{\hbar}\left(p_{1}, p_{2}\right)=\exp \left(-\pi \hbar \gamma\left(p_{1}, p_{2}\right)\right) .
$$

Using this, define a new associative product $\star_{\hbar}$ on $\mathcal{S}(\hat{T})$,

$$
\left(\psi \star_{\hbar} \phi\right)(p)=\sum_{p_{1}+p_{2}=p} \psi\left(p_{1}\right) \phi\left(p_{2}\right) \sigma_{\hbar}\left(p_{1}, p_{2}\right) .
$$

This is precisely the smooth noncommutative torus $A_{\sigma_{\hbar}}^{\infty}$.

The norm $\|\cdot\|_{\hbar}$ is defined to be the operator norm for the action of $\mathcal{S}(\hat{T})$ on $L^{2}(\hat{T})$ given by $\star_{\hbar}$. Via the Fourier transform, carry this structure back to $C^{\infty}(T)$, to obtain the smooth noncommutative torus as a strict deformation quantization of $C^{\infty}(T)$, 15, with respect to the translation action of $T$.

Example 6.2. We next generalize the above to the case of principal torus bundles $q: Y \rightarrow X$ of rank equal to $n$. Note that fibrewise smooth functions on $Y$ decompose as a direct sum,

$$
\begin{aligned}
C_{\text {fibre }}^{\infty}(Y) & =\widehat{\bigoplus}_{\alpha \in \hat{T}} C_{\text {fibre }}^{\infty}\left(X, \mathcal{L}_{\alpha}\right) \\
\phi & =\sum_{\alpha \in \hat{T}} \phi_{\alpha}
\end{aligned}
$$

where $C_{\text {fibre }}^{\infty}\left(X, \mathcal{L}_{\alpha}\right)$ is defined as the subspace of $C_{\text {fibre }}^{\infty}(Y)$ consisting of functions which transform under the character $\alpha \in \hat{T}$, and where $\mathcal{L}_{\alpha}$ denotes the associated line bundle $Y \times_{\alpha} \mathbf{C}$ over $X$. That is, $\phi_{\alpha}(y t)=\alpha(t) \phi_{\alpha}(y), \forall y \in Y, t \in T$. The direct sum is completed in such a way that the function $\hat{T} \ni \alpha \mapsto\left\|\phi_{\alpha}\right\|_{\infty} \in \mathbf{R}$ is in $\mathcal{S}(\hat{T})$. In this interpretation of $C_{\text {fibre }}^{\infty}(Y)$, it is easy to extend to this case, the explicit deformation quantization given in the previous example, which we now briefly outline. For $\phi, \psi \in C_{\text {fibre }}^{\infty}(Y)$, define a new associative product $\star_{\hbar}$ on $C_{\text {fibre }}^{\infty}(Y)$ as follows. For $y \in Y, \alpha, \alpha_{1}, \alpha_{2} \in \hat{T}$, let

$$
\left(\psi \star_{\hbar} \phi\right)(y, \alpha)=\sum_{\alpha_{1} \alpha_{2}=\alpha} \psi\left(y, \alpha_{1}\right) \phi\left(y, \alpha_{2}\right) \sigma_{\hbar}\left(q(y) ; \alpha_{1}, \alpha_{2}\right),
$$

using the notation $\psi\left(y, \alpha_{1}\right)=\psi_{\alpha_{1}}(y)$ etc., and where $\sigma_{\hbar} \in C_{b}\left(X, Z^{2}(\hat{T}, \mathbf{T})\right)$ is a continuous family of bicharacters of $\hat{T}$ such that $\sigma_{0}=1$, which is part of the data that we start out with. We remark that one way to get such a $\sigma_{\hbar}$ is to choose a continuous family skew-symmetric forms on $\hat{T}, \gamma: X \longrightarrow Z^{2}(\hat{T}, \mathbf{R})$, and define $\sigma_{\hbar}=\exp (-\pi \hbar \gamma)$. In the case of the principal torus bundle $Y$, we note that the vertical tangent bundle of $Y$ has a Poisson structure, i.e. $\gamma \in \bigwedge^{2} T^{v e r t} Y$, which can be naturally interpreted as a continuous family of symplectic structures along the fibre, that is, $\gamma$ is of the sort considered just previously. We denote the deformed algebra by $C_{\text {fibre }}^{\infty}(Y)_{\hbar}$, and using $\$ 3$ we can realize it as a parametrised strict 
deformation quantization of $C_{\text {fibre }}^{\infty}(Y)$. Since the construction is $T$-equivariant, $C_{\text {fibre }}^{\infty}(Y)_{\hbar}$ has a $T$-action that is induced from the given $T$-action on $C_{\text {fibre }}^{\infty}(Y)$.

Example 6.3. We next consider the example which was one of the inspirations for Theorem 5.1 Although it is a special case of the previous example, and is probably also treated elsewhere, nevertheless we think that it is worthwhile to treat in our context. Consider a 3-dimensional torus, which we write as $S^{1} \times T$, where $T$ is a two dimensional torus. Let $\{\cdot, \cdot\}$ denote the Poisson bracket on $S^{1} \times T$ coming from $T$ and trivial on $S^{1}$. Then this Poisson bracket is invariant under the $T$ action on $S^{1} \times T$, where $T$ acts trivially on $S^{1}$ and via translation on itself. Here the fibres are $T$. As in the example above, we construct a strict deformation quantization of $C_{\text {fibre }}^{\infty}\left(S^{1} \times T\right)$. Taking the partial Fourier transform in the $T$-variables, we obtain an isomorphism between $C_{\text {fibre }}^{\infty}\left(S^{1} \times T\right)$ and $\mathcal{S}_{\text {fibre }}\left(S^{1} \times \hat{T}\right)$. In the notation of the previous example, for $\phi, \psi \in \mathcal{S}_{\text {fibre }}\left(S^{1} \times \hat{T}\right)$, define

$$
\left(\psi \star_{\hbar} \phi\right)(y, p)=\sum_{p_{1}+p_{2}=p} \psi\left(y, p_{1}\right) \phi\left(y, p_{2}\right) \sigma_{\hbar}\left(y ; p_{1}, p_{2}\right) .
$$

where $\sigma_{\hbar}: S^{1}=\mathbf{R} / \mathbf{Z} \longrightarrow H^{2}(\hat{T}, \mathbf{T}) \cong \mathbf{T}$ is the family of bicharacters of $\hat{T}$ given by

$$
\sigma_{\hbar}\left(y ; p_{1}, p_{2}\right)=\exp \left(-\pi \hbar y \gamma\left(p_{1}, p_{2}\right)\right) .
$$

Here $\gamma$ is defined as in Example 6.1. This gives us a family of smooth noncommutative tori, that is,

$$
\mathcal{S}_{\text {fibre }}\left(S^{1} \times \hat{T}\right)_{\hbar}=\int_{y \in S^{1}} A_{\sigma_{\hbar}(y)}^{\infty}
$$

which in turn can be identified with (when $\hbar=1$ ) the fibrewise Schwartz subalgebra of the 3-dimensional integer Heisenberg group, Heisz. That is, the norm closure of $\mathcal{S}_{\text {fibre }}\left(S^{1} \times \hat{T}\right)_{\hbar=1}$ is isomorphic to $C^{*}\left(\right.$ Heis $\left._{\mathbf{Z}}\right)$.

On the other hand, using the results of $\oint 3$, we see that $\mathcal{S}_{\text {fibre }}\left(S^{1} \times \hat{T}\right)_{\hbar}$ is a parametrised strict deformation quantization of $C_{\text {fibre }}^{\infty}\left(S^{1} \times T\right)$.

Example 6.4. Motivated by Theorem 5.1 and an example in [15, we define noncommutative non-principal torus bundles as follows. Let $\rho: \pi_{1}(X) \rightarrow \operatorname{Sp}(2 n, \mathbf{Z})$ be a representation of the fundamental group, $T=\mathbf{R}^{2 n} / \mathbf{Z}^{2 n}$ be the torus and $q: Y_{\rho} \rightarrow X$ be the non-principal torus bundle given by $Y_{\rho}=(\widetilde{X} \times T) / \pi_{1}(X)$, where we observe that the symplectic group is a subgroup of the automorphism group of $T$ and $\Gamma=\pi_{1}(X)$ acts on $T$ via $\rho$ and on the universal cover $r: \widetilde{X} \longrightarrow X$ via deck transformations.

Let $\{\cdot, \cdot\}$ denote the Poisson bracket on $\widetilde{X} \times T$ coming from $T$ and trivial on $\tilde{X}$. Then this Poisson bracket is invariant under the $T$ action on $\widetilde{X} \times T$, therefore descending to a Poisson bracket on $\widetilde{X}$, denoted by the same symbol. As in the example above, we construct a strict deformation quantization of $C_{\text {fibre }}^{\infty}(\widetilde{X} \times T)$, which is the algebra of continuous functions on $\tilde{X} \times T$ that are smooth along the fibres. Taking the partial Fourier transform in the $T$-variables, we obtain an isomorphism between $C_{\text {fibre }}^{\infty}(\widetilde{X} \times T)$ and $\mathcal{S}_{\text {fibre }}(\widetilde{X} \times \hat{T})$. In the notation of the previous example, for $\phi, \psi \in \mathcal{S}_{\text {fibre }}(\widetilde{X} \times \hat{T})$, define

$$
\left(\psi \star_{\hbar} \phi\right)(y, p)=\sum_{p_{1}+p_{2}=p} \psi\left(y, p_{1}\right) \phi\left(y, p_{2}\right) \sigma_{\hbar}\left(r(y) ; p_{1}, p_{2}\right) .
$$


where $\sigma_{\hbar}: X \longrightarrow Z^{2}(\hat{T}, \mathbf{T})$ is a continuous family of bicharacters of $\hat{T}$, which is part of the data that we start out with.

Then transporting this structure back to $C_{\text {fibre }}^{\infty}(\widetilde{X} \times T)$ gives a strict deformation quantization such that $\Gamma=\pi_{1}(X)$ acts properly on it, cf. [15. We denote the deformed algebra as $C_{\text {fibre }}^{\infty}(\tilde{X} \times T)_{\hbar}$. The fixed point subalgebra $C_{\text {fibre }}^{\infty}(\widetilde{X} \times T)_{\hbar}^{\Gamma}$ of the deformed algebra is then the desired parametrised strict deformation quantization, $C_{\text {fibre }}^{\infty}(\tilde{X} \times T)_{\hbar}^{\Gamma}=C_{\text {fibre }}^{\infty}\left(Y_{\rho}\right)_{\sigma_{\hbar}}$, where we note that $C_{\text {fibre }}^{\infty}(\widetilde{X} \times T)^{\Gamma}=C_{\text {fibre }}^{\infty}\left(Y_{\rho}\right)$. This is our definition of a noncommutative non-principal torus bundle. To summarize, it is determined by two pieces of data:

- $\rho \in \operatorname{Hom}\left(\pi_{1}(X), \operatorname{Sp}(2 n, \mathbf{Z})\right)$;

- $\sigma \in C\left(X, Z^{2}(\hat{T}, \mathbf{T})\right)$, that is, a continuous family of bicharacters of $\hat{T}$.

\section{Appendix A. Factors of Automorphy}

Appendix $\mathrm{C}$ to 1 introduced a method for lifting algebra bundles to a contractible universal cover and encoding information about the Dixmier-Douady class in a factor of automorphy $\widehat{j}$. This also fits into a parametrised deformation picture, but with the further generalisation that the group $\Gamma$ now acts on the parameter space $X$. The cocycle $\hat{j}$ for a lifting can be reconstructed from the Dixmier-Douady class $\delta \in H^{3}(X, \mathbf{Z}) \sim H^{3}(\Gamma, \mathbf{Z})$, by first finding $\widehat{\tau}\left(k_{1}, k_{2}, x\right)\left(k_{1}, k_{2} \in \Gamma, x \in X\right)$ with $d \widehat{\tau}=\delta$, defining $\tau=\exp (2 \pi i \widehat{\tau})$, and then finding $\widehat{j}(k, x)$ satisfying

$$
\widehat{j}\left(k_{1}, k_{2} x\right) \widehat{j}\left(k_{2}, x\right)=\tau\left(k_{1}, k_{2}, x\right) \widehat{j}\left(k_{1} k_{2}, x\right),
$$

which can be achieved by a modified $\tau$-inducing construction, which gives $\widehat{j}$ in terms of $\tau$.

We know that $\tau$ is a $C_{0}(X)$-valued cocycle satisfying the cocycle condition

$$
\tau\left(k_{1} k_{2}, k_{3}\right) \alpha_{k_{3}}^{-1}\left[\tau\left(k_{1}, k_{2}\right)\right]=\tau\left(k_{1}, k_{2} k_{3}\right) \tau\left(k_{2}, k_{3}\right)
$$

where $\alpha$ just gives the translation action on $C_{0}(X)$, and similarly suppressing the $X$-dependence in $\widehat{j}$ allows us to rewrite its cocycle condition as

$$
\alpha_{k_{2}}^{-1}\left[\widehat{j}\left(k_{1}\right)\right] \widehat{j}\left(k_{2}\right)=\tau\left(k_{1}, k_{2}\right) \widehat{j}\left(k_{1} k_{2}\right) .
$$

The cocycle condition on $\tau$ can also be written as

$$
\alpha_{k_{3}}\left[\tau\left(k_{1} k_{2}, k_{3}\right)\right] \tau\left(k_{1}, k_{2}\right)=\alpha_{k_{3}}\left[\tau\left(k_{1}, k_{2} k_{3}\right)\right] \alpha_{k_{3}}\left[\tau\left(k_{2}, k_{3}\right)\right]
$$

so setting $U\left(k_{1}\right): k \mapsto \alpha_{k}\left[\tau\left(k_{1}, k\right)\right]$ we get

$$
U\left(k_{1} k_{2}\right)\left(k_{3}\right) \tau\left(k_{1}, k_{2}\right)=\alpha_{k_{2}}^{-1}\left[U\left(k_{1}\right)\left(k_{2} k_{3}\right)\right] U\left(k_{2}\right)\left(k_{3}\right) .
$$

We can lift the automorphism $\alpha_{k_{2}}$ to

$$
\left.\widetilde{\alpha}_{k_{2}}^{-1}\left[U\left(k_{1}\right)\right]\left(k_{3}\right)\right]=\alpha_{k_{2}}^{-1}\left[U\left(k_{1}\right)\left(k_{2} k_{3}\right)\right]
$$

and then

$$
U\left(k_{1} k_{2}\right) \tau\left(k_{1}, k_{2}\right)=\widetilde{\alpha}_{k_{2}}^{-1}\left[U\left(k_{1}\right)\right] U\left(k_{2}\right),
$$

the type of cocycle condition to be satisfied by $\widehat{j}$.

To compare these with the Landstad-Kasprzak construction we take $\Gamma=\widehat{V}$, $\widehat{\rho}$ the left translation $\left(L_{k} f\right)(x)=f\left(k^{-1} x\right)$. Now we think of $\widehat{j}$ as a map from $K$ to unitary multipliers on $C_{0}(X)$, and take $U(k): x \mapsto \widehat{j}\left(k, k^{-1} x\right)$, noting that the cocycle condition $\widehat{j}\left(k_{1}, k_{2} x\right) \widehat{j}\left(k_{2}, x\right)=\tau\left(k_{1}, k_{2}, x\right) \widehat{j}\left(k_{1} k_{2}, x\right)$ gives

$$
U\left(k_{1}\right) \widehat{\rho}\left(k_{1}\right)\left[U\left(k_{2}\right)\right]=\tau\left(k_{1}, k_{2}\right) U\left(k_{1} k_{2}\right)
$$


precisely the condition arising in deformation (though the new ingredient is that $K$ acts on the $X$ argument of $\tau$ ).

We note also that the predual algebra in the Landstad theory is the generalised fixed point algebra $\mathcal{A}=\mathcal{B}^{\widehat{\rho}}$.

\section{REFERENCES}

[1] P. Bouwknegt, K.C. Hannabuss, and V. Mathai, $C^{*}$-algebras in tensor categories, Clay Mathematics Proceedings. 12 (2009) 39 pages, (in press). math.QA/0702802

[2] A. Connes, $C^{*}$-algèbres et géometrie différentielle, C.R. Acad. Sci. Paris, Ser. A-B, 290, (1980) no. 13, 599-604.

[3] S. Echterhoff, R. Nest, and H. Oyono-Oyono, Principal non-commutative torus bundles, Proc. London Math. Soc. (3) 99, (2009) 1-31.

[4] S. Echterhoff and D. P. Williams, Crossed products by $C_{0}(X)$-actions, J. Funct. Anal. 158 (1998) no. 1, 113-151.

[5] K.C. Hannabuss, Representations of nilpotent locally compact groups, J. Funct. Anal. 34 (1979) no. 1, 146-165.

[6] A. an Huef, I. Raeburn, and D.P. Williams, Functoriality of Rieffel's generalised fixed point algebras for proper actions, arXiv:0909.2860.

[7] S. Kaliszewski and J. Quigg, Categorical Landstad duality for actions, Indiana Math. J. 58 (2009) 415-441.

[8] G. Kasparov, Equivariant K-theory and the Novikov conjecture, Invent. Math. 91, (1988) 147-201.

[9] P. Kasprzak, Rieffel deformation via crossed products, J. Funct. Anal. 257 (2009) 1288-1332.

[10] A. Kleppner, Multipliers on abelian groups, Math. Ann. 158 (1965) 11-34.

[11] M.B. Landstad, Duality theory for covariant systems, Trans. Amer. Math. Soc. 248 (1979) 223-267.

[12] M.B. Landstad, Quantization arising from abelian subgroups, Internat. J. Math. 5 (1994) 897-936.

[13] V. Mathai and J. Rosenberg, T-duality for torus bundles via noncommutative topology, Commun. Math. Phys., 253 no. 3 (2005) 705-721. hep-th/0401168.

[14] V. Mathai and J. Rosenberg, T-duality for torus bundles with H-fluxes via noncommutative topology, II: the high-dimensional case and the T-duality group, Adv. Theor. Math. Phys., 10 no. 1 (2006) 123-158. hep-th/0508084.

[15] M.A. Rieffel, Deformation quantization for actions of $\mathbf{R}^{d}$, Memoirs of the Amer. Math. Soc. 106 (1993), no. 506, 93 pp.

[16] M.A. Rieffel, Quantization and $C^{*}$-algebras, Contemporary Math. 167, (1994), 67-97.

[17] M.A. Rieffel, Applications of strong Morita equivalence to transformation group $C^{*}$-algebras, Proceedings of Symposia in Pure Mathematics, 38 (1982) Part I, 299-310.

(Keith Hannabuss) Mathematical Institute, 24-29 St. Giles', Oxford, OX1 3LB, And Balliol College, Oxford, OX1 3BJ, England

E-mail address: kch@balliol.oxford.ac.uk

(Varghese Mathai) Department of Pure Mathematics, University of Adelaide, AdeLAIDE, SA 5005, Australia

E-mail address: mathai.varghese@adelaide.edu.au 